\title{
Optical nanoantenna input impedance
}

\author{
Yuancheng Xu ${ }^{1}$, Eric Tucker ${ }^{2}$, Glenn Boreman ${ }^{2}$, Markus B. Raschke ${ }^{3}$, and Brian A. Lail ${ }^{1 \star}$ \\ ${ }^{1}$ Department of Electrical Engineering, Florida Institute of Technology, Melbourne, Florida, USA \\ ${ }^{2}$ Department of Physics and Optical Science, University of North Carolina at Charlotte, North Carolina, USA \\ ${ }^{3}$ Department of Physics, Department of Chemistry, and JILA, University of Colorado, Colorado, USA \\ e-mail: blail@fit.edu
}

\section{Supporting Information}

\section{S.1 Equation for measurement data fitting}

The magnitude of electric field along the transmission line represents a standing wave, which results from the optical wave coupling into the coplanar strip (CPS) from the dipole antenna end and the load antenna end. This superposition was described by a geometric-series expansion to account for all the reflections at the impedance mismatched loads:

$$
\begin{gathered}
e_{0}=e^{(-(\alpha+\mathrm{i} \beta) \mathrm{L})}, \\
e_{1}=e^{(-(\alpha+\mathrm{i} \beta)(\mathrm{L}-\mathrm{x}))}, \\
e_{2}=e^{(-(\alpha+\mathrm{i} \beta) \mathrm{x})}, \\
E_{Z}(\mathrm{x})=E_{d}\left(e_{0} e_{1} \Gamma_{L}+\frac{\left(e_{0}^{3}\right)\left(\Gamma_{L}^{2}\right) \Gamma_{d} e_{1}}{\left(1-\left(e_{0}^{2}\right) \Gamma_{d} \Gamma_{L}\right)}+e_{2}+\frac{\left(e_{0}^{2}\right) \Gamma_{L} \Gamma_{d} e_{2}}{\left(1-\left(e_{0}^{2}\right) \Gamma_{d} \Gamma_{L}\right)}\right)+E_{L}\left(e_{1}+\frac{\left(e_{0}^{2}\right) \Gamma_{d} \Gamma_{L} e_{1}}{\left(1-\left(e_{0}^{2}\right) \Gamma_{d} \Gamma_{L}\right)}+e_{0} e_{2} \Gamma_{d}+\frac{\left(e_{0}^{3}\right) \Gamma_{L}\left(\Gamma_{d}^{2}\right) e_{2}}{\left(1-\left(e_{0}^{2}\right) \Gamma_{d} \Gamma_{L}\right)}\right)
\end{gathered}
$$

where $\Gamma_{\mathrm{L}}$ is the reflection coefficient at the load terminal (for example: a folded dipole), and $\Gamma_{\mathrm{d}}$ is the reflection at the single dipole terminal. $\Gamma_{\mathrm{d}}$ is determined in two ways; 1) using a lumped-port excitation, and 2) by fitting the standing wave on a CPS loaded with identical dipoles on either end, with good agreement and with values validated against prior results in reference [25]. $E_{d}$ and $E_{L}$ are the magnitude at dipole side and load side. $L$ is the length of the transmission line. The origin is at the dipole end $(\mathrm{x}=0)$ similar to the paper. The attenuation constant $\alpha$ was extracted from the decaying complex field amplitude as a function of position along an electrically long CPS predicted 
through full-wave analysis and the phase constant $\beta$ was determined from the spatial periodicity in the wave function, respectively:

$$
\begin{aligned}
& \beta=\frac{2 \pi}{\lambda} \\
& \alpha=\frac{20 \log _{10}(E 1 / E 2)}{8.69 d}
\end{aligned}
$$

where $\mathrm{d}$ is the distance between E1 and E2. The attenuation and phase constant should be averaged by calculating through the whole length of CPS.

The impedance of the antenna under test (AUT) was determined from the reflection coefficient, $\Gamma_{\mathrm{L}}$, expressed as:

$$
Z_{L}=\left(\frac{1+\Gamma_{L}}{1-\Gamma_{L}}\right) Z_{0}
$$

where $Z_{0}$ is the characteristic impedance of the CPS, $\Gamma_{L}$ is the reflection coefficient at the load terminal which was determined from the fitting equation (S4) and $\mathrm{Z}_{\mathrm{L}}$ is the impedance of the load antenna.

The characteristic impedance of CPS, which exhibits a high confinement associated with transmission mode here, was determined by changing the open-circuit and short-circuit load with expression:

$$
Z_{\text {in }}=Z_{o}\left(\frac{Z_{L} \cosh (\gamma L)+Z_{0} \sinh (\gamma L)}{Z_{0} \cosh (\gamma L)+Z_{L} \sinh (\gamma L)}\right)
$$

where $\mathrm{L}$ is the length of CPS, $\gamma=\alpha+\mathrm{j} \beta, Z_{L}$ is load impedance and $Z_{0}$ is input impedance of CPS. In the open-circuit mode operation, $Z_{L}$ goes to a very large number, $Z_{o c}=Z_{o}\left(\frac{\cosh (\gamma L)}{\sinh (\gamma L)}\right)$, while in the short-circuit mode operation, $Z_{L}$ equals zero, $Z_{s c}=Z_{o}\left(\frac{\sinh (\gamma L)}{\cosh (\gamma L)}\right)$. The input impedance of CPS can be achieved from following:

$$
Z_{0}=\sqrt{\left(Z_{s c} Z_{o c}\right)}
$$

where $Z_{s c}$ is the input impedance when $Z_{L}$ is a short-circuit load and $Z_{o c}$ is the input impedance when $Z_{L}$ is an opencircuit load. 


\section{S.2 Numerical method of driven model in HFSS}

Ansys high frequency structural simulator (HFSS) is a commercial finite element method solver for electromagnetic structures (http://www.ansys.com). The driven model in HFSS was implemented to compute the input impedance of nanoantennas directly, by driving the nanoantenna at its feed gap with an optical source. Antenna impedance, $\mathrm{Z}_{\mathrm{A}}$, can be defined as the ratio of the effective voltage across the antenna arms and the optical displacement current flowing through the gap. Fig. S1 gives an example of feed-gap excitation for the folded dipole antenna in HFSS.

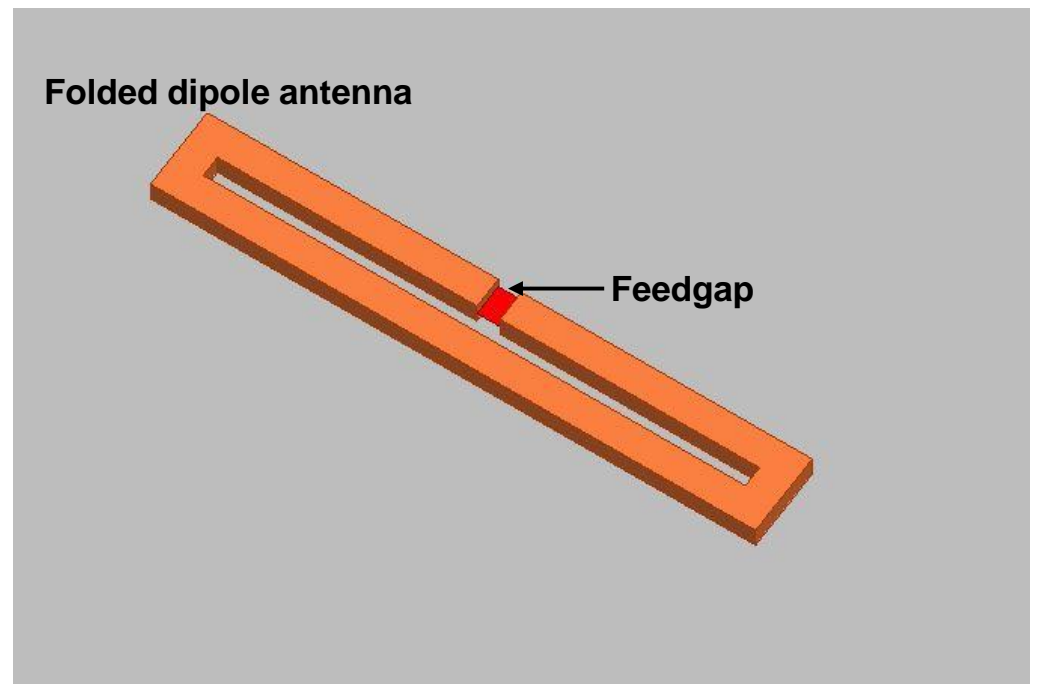

Figure S1| Folded dipole antenna with a feed gap excitation in HFSS 
S.3 Analytical fitting to the measured data of double-folded and triple-folded dipole antennas
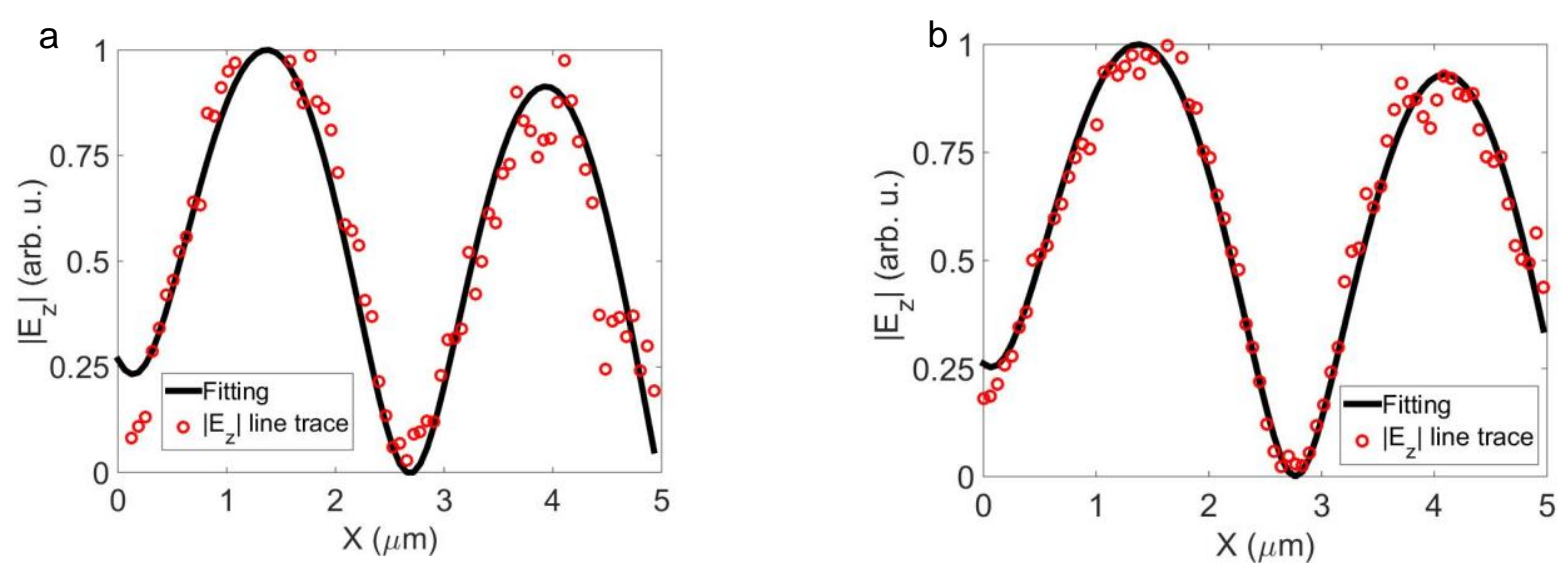

Figure S2| Analytical fitting to the measured data for the (a) doubled- and (b) triple-folded dipole antennas 